\title{
ORIENTATIONS ON 2-VECTOR BUNDLES AND DETERMINANT GERBES
}

\author{
THOMAS KRAGH*
}

\begin{abstract}
In a paper from 2009, a half magnetic monopole was discovered by Ausoni, Dundas, and Rognes. This describes an obstruction to the existence of a continuous map $K(k u) \rightarrow B\left(k u^{*}\right)$ with determinant like properties. This magnetic monopole is in fact an obstruction to the existence of a map from $K(k u)$ to $K(\mathbf{Z}, 3)$, which is a retract of the natural map $K(\mathbf{Z}, 3) \rightarrow K(k u)$; and any sensible definition of determinant like should produce such a retract. In this paper we describe this obstruction precisely using monoidal categories. By a result from 2011 by Baas, Dundas, Richter and Rognes $K(k u)$ classifies 2-vector bundles. We thus define the notion of oriented 2vector bundles, which removes the obstruction by the magnetic monopole. We use this to define an oriented K-theory of 2-vector bundles with a lift of the natural map from $K(\mathrm{Z}, 3)$. It is then possible to define a retraction of this map and since $K(\mathrm{Z}, 3)$ classifies complex gerbes we call this a determinant gerbe map.
\end{abstract}

\section{Introduction}

Let $\mathscr{V}$ be the category with objects $C^{n}$ for $n \geq 0$ and morphisms the complex linear isomorphisms. This is a symmetric bimonoidal (in fact bipermutative) category with respect to $\oplus$ and $\otimes$. So, as in [5] one can form the category $\mathrm{M}_{n}(\mathscr{V})$ with objects being $n$ by $n$ matrices of objects in $\mathscr{V}$ and morphisms the entry-wise isomorphisms. The category $\mathrm{M}_{n}(\mathscr{V})$ has a monoidal product given by the usual matrix product with addition and multiplication replaced by $\oplus$ and $\otimes$ respectively. By taking dimension entry-wise we get a map from the objects of $M_{n}(\mathscr{V})$ to $M_{n}\left(\mathrm{~N}_{0}\right) \subset M_{n}(\mathrm{Z})$ (Standard matrices with [non-negative] integer entries). We define the weakly invertible matrices $\mathrm{Gl}_{n}(\mathscr{V})$ as the full sub-category of those objects which map to invertible matrices in $\mathrm{M}_{n}(\mathrm{Z})$. In [5] the authors defined charted 2-vector bundles of rank $n$. They proved that $\left|\mathscr{B} \mathrm{Gl}_{n}(\mathscr{V})\right|$ defines a classifying space for equivalence classes of charted 2vector bundles of rank $n$. Here $\mathscr{B}$ is a categorical bar construction and $|\cdot|$ denotes taking nerve and geometric realization.

The disjoint union $\bigsqcup_{n \geq 0}\left|\mathscr{B} \mathrm{Gl}_{n}(\mathscr{V})\right|$ of these classifying spaces is a monoid under block sum operation. In [5] the authors conjectured that the group

\footnotetext{
* The author was funded by the Topology in Norway Project, and would like to thank John Rognes and Bjørn Jahren for many conversations on the subject.

Received 15 July 2011, in final form 4 October 2012.
} 
completion

$$
K(\mathscr{V})=\Omega B\left(\coprod_{n \geq 0}\left|\mathscr{B} \mathrm{Gl}_{n}(\mathscr{V})\right|\right) \simeq \mathrm{Z} \times\left|\mathscr{B} \mathrm{Gl}_{\infty}(\mathscr{V})\right|^{+}
$$

is equivalent as an infinite loop space to $K(k u)$. This conjecture was proven by the authors in [4]. Here $k u=\mathrm{Z} \times B \mathrm{U}$ represents connective complex K-theory. The K-theory $K(k u)$ may be defined as the algebraic K-theory of $k u$ using the work of Elmendorf and Mandell in [7].

A complex gerbe (defined in [6]) is equivalent to a charted 2-vector bundle of rank 1 . These are classified by the Eilenberg-Maclane space $K(\mathrm{Z}, 3)$. Indeed, $\left|\mathscr{B} \mathrm{Gl}_{1}(\mathscr{V})\right| \simeq K(\mathrm{Z}, 3)$. So, the inclusion $\mathrm{Gl}_{1}(\mathscr{V}) \rightarrow \mathrm{Gl}_{n}(\mathscr{V})$ (using block sum with the $n-1$ by $n-1$ unit) defines a canonical map

$$
K(\mathrm{Z}, 3) \simeq\left|\mathscr{B} \mathrm{Gl}_{1}(\mathscr{V})\right| \rightarrow\left|\mathscr{B} \mathrm{Gl}_{n}(\mathscr{V})\right| .
$$

In [1], the authors proved that if $n$ is large enough then the image of the canonical generator of $\pi_{3}(K(\mathrm{Z}, 3))$ under the above map is divisible by two (modulo torsion). As noted in [1] this means that no retraction back to $K(\mathrm{Z}, 3)$ of the map in Equation (1) exists. This provides and obstruction to defining a determinant-like map

$$
\left|\mathscr{B} \mathrm{Gl}_{n}(\mathscr{V})\right| \rightarrow\left|\mathscr{B} \mathrm{Gl}_{1}(\mathscr{V})\right| .
$$

Indeed, any sensible definition here of determinant-like would yield a retraction.

In light of the result that $K(\mathscr{V}) \simeq K(k u)$, and since a determinant really should be a signed sum of products of the entries, a more natural recipient of a determinant map would be $\left|\mathscr{B} \mathrm{Gl}_{1}(\overline{\mathscr{V}})\right|$. Here $\overline{\mathscr{V}}$ is the ring completion constructed in [2], which adds inverse objects with respect to $\oplus$ (up to an equivalence that we will ignore) while retaining the symmetric bimonoidal structure. By Theorem 1.3 in [2] we have $|\overline{\mathscr{V}}| \simeq \mathrm{Z} \times B \mathrm{U}=k u$, and so $\left|\mathrm{Gl}_{1}(\overline{\mathscr{V}})\right| \simeq\{ \pm 1\} \times B \mathrm{U}=k u^{*}$, implying that

$$
\left|\mathscr{B} \mathrm{Gl}_{1}(\overline{\mathscr{V}})\right| \simeq B_{\otimes}\left(k u^{*}\right) .
$$

However, since $B_{\otimes} k u^{*}$ has $K(\mathrm{Z}, 3) \simeq B_{\otimes} B \mathrm{U}(1)$ as a retract (see Corollary 6.2) the magnetic monopole is also an obstruction for the existence of this more natural notion of determinant. Indeed, such a determinant composed with the retraction to $K(\mathrm{Z}, 3)$ would yield a retraction of Equation (1).

The reader might wonder at this point why the existence of the ring completed symmetric bimonoidal category $\overline{\mathscr{V}}$ does not immediately give a determinant map by making a choice of the orderings of the terms in the usual determinant formula for commutative rings. The reason for this is a little subttle. 
Indeed, By making a choice of inverse for $C^{1}$ with respect to $\oplus$ in $\overline{\mathscr{V}}$ and tensoring with this as a choice of negative for all other objects $A$ one may, indeed, define a functor

$$
\mathrm{Gl}_{n}(\overline{\mathscr{V}}) \rightarrow \mathrm{Gl}_{1}(\overline{\mathscr{V}})
$$

However, when trying to prove the product formula one needs to "coherently cancel terms", which in this case is not possible (proved by the obstruction). Indeed, one gets into trouble when making choices to pair up terms and canceling. This cannot be done in a way that allows for the definition of the natural transformation which are supposed to intertwine the functor with the associators and the products. So, it is not possible to construct this as a monoidal functor, and subsequently the functor does not deloop.

In this paper, we describe the obstruction given by the half magnetic monopoles in the framework of monoidal categories. Using this, we define a natural notion of an orientation on a 2-vector bundle. Furthermore, we define a monoidal category $\mathrm{O}\left(\mathscr{V}^{n}\right)$ such that $\left|\mathscr{B O}\left(\mathscr{V}^{n}\right)\right|$ classifies oriented 2-vector bundles. There is a natural, forgetful, and strict monoidal functor from $\mathrm{O}\left(\mathscr{V}^{n}\right)$ to $\mathrm{Gl}_{n}(\mathscr{V})$ inducing a map of classifying spaces

$$
\left|\mathscr{B} \mathrm{O}\left(\mathscr{V}^{n}\right)\right| \rightarrow\left|\mathscr{B} \mathrm{Gl}_{n}(\mathscr{V})\right| .
$$

We then describe the precise obstruction to lifting a map $f: X \rightarrow\left|B \mathrm{Gl}_{n}(\mathscr{V})\right|$ to the oriented "cover". This is given by a characteristic class in $H^{3}(X, \mathrm{Z} / 2 \mathrm{Z})$. We then describe a canonical lift of the inclusion of gerbes in Equation (1). We finally construct a determinant gerbe functor from $\mathrm{O}\left(\mathscr{V}^{n}\right)$ back to $\mathrm{Gl}_{1}(\mathscr{V})$. The two maps are easily seen to define an inclusion with a retraction

$$
K(\mathrm{Z}, 3) \rightarrow\left|B \mathrm{O}\left(\mathscr{V}^{n}\right)\right| \rightarrow K(\mathrm{Z}, 3) .
$$

The notion of orientations then makes it possible to define an oriented version of K-theory of $\mathscr{V}$ as

$$
K^{\text {or }}(\mathscr{V})=\Omega B\left(\coprod_{n \in \mathbb{N}}\left|\mathscr{B} \mathrm{O}\left(\mathscr{V}^{n}\right)\right|\right) \simeq \mathrm{Z} \times\left|\mathscr{B O}\left(\mathscr{V}^{\infty}\right)\right|^{+} .
$$

This comes with a canonical map to $K(\mathscr{V}) \simeq K(k u)$.

In Section 2, we properly define the bipermutative category $\mathscr{V}$. We also define a related bipermutative category $\mathscr{L}$. Indeed, in light of Lemma 2.6, one may heuristically think of $\mathscr{L}$ as a categorical model for the second Postnikov section of $\mathscr{V}$ group-completed with respect to $\oplus$ (i.e. a categorical model for the second Postnikov section of $|\overline{\mathscr{V}}|$ ). 
In Section 3, we describe the category of matrices $\mathrm{M}_{n}(\mathscr{C})$ in a bipermutative category $\mathscr{C}$. We the define the weakly invertible sub-category $\mathrm{Gl}_{n}(\mathscr{V})$ of $\mathrm{M}_{n}(\mathscr{V})$. We also construct determinant-like functors to $\mathscr{L}$. These turn out not to be monoidal even though they preserve the product.

In Section 4, we define $\mathrm{O}\left(\mathscr{V}^{n}\right)$, which is an oriented version of $\mathrm{Gl}_{n}(\mathscr{V})$. The structure in $\mathrm{O}\left(\mathscr{V}^{n}\right)$ is inspired by the determinant-like functors from Section 3. We are then able to define determinant-like monoidal functors from $\mathrm{O}\left(\mathscr{V}^{n}\right)$ to $\mathrm{Gl}_{1}(\mathscr{V})$.

In Section 5, we recall the definition of charted 2-vector bundles and define oriented charted 2-vector bundles. We then describe the obstruction for a 2vector bundle to be oriented as a characteristic class in the third cohomology class of the base.

In Section 6, we define (charted) gerbes. Then, we lift the inclusion of gerbes to oriented 2-vector bundles. Using the monoidal functor from Section 4 we now obtain a splitting back to gerbes as in Equation (3).

In Section 7, we generalize the block sum construction to the oriented categories. This makes it possible to define $K^{\text {or }}(\mathscr{V})$ as in Equation (4).

An earlier version on arXiv.org includes a section on connective structures. This has been removed due to an error.

\section{The Bipermutative Categories $\mathscr{V}$ and $\mathscr{L}$}

A bipermutative category $(\mathscr{C}, \oplus, \otimes, 0,1)$ is a symmetric bimonoidal category in which the following operations are strict;

- the unit 0 for $\oplus$,

- the unit 1 for $\otimes$,

- associativity for both $\oplus$ and $\otimes$, and

- the right distributive law $(A \oplus B) \otimes C=(A \otimes C) \oplus(B \otimes C)$.

Here the equality means that the two functors representing the operations are equal. All the coherency isomorphisms in a bipermutative category are generated by the two twists $\underline{\mathrm{c}}^{\oplus}$ and $\underline{\mathrm{c}}^{\otimes}$. Indeed, coherencies for the left distributive law are given by

$$
C(A \oplus B) \stackrel{\stackrel{\mathrm{c}}{C}, A \oplus B^{\otimes}}{\longrightarrow}(A \oplus B) C=A C \oplus B C \stackrel{\mathrm{c}_{A, C}^{\otimes} \oplus \underline{\mathrm{c}}_{B, C}^{\otimes}}{\longrightarrow} C A \oplus C B .
$$

Here we have omitted the $\otimes$ 's for brevity. For a complete list of the diagrams involving $\underline{\mathrm{c}}^{\otimes}$ and $\underline{\underline{c}}^{\oplus}$ that need to commute in a bipermutative category we refer to [7]. 
Definition 2.1. Let $\Sigma$ be the category with

- objects the sets $\mathbf{n}=\{1, \ldots, n\}$ for $n \in \mathrm{N}_{0}$ and

- morphisms the permutations $\sigma_{n} \in \Sigma_{n}$ of $\mathbf{n}$.

Note that $\mathrm{N}_{0}=\{0,1, \ldots\}$ and $\mathbf{0}=\emptyset$.

The sum functor,

$$
\oplus: \Sigma \times \Sigma \rightarrow \Sigma
$$

is defined by concatenation. Indeed, on objects it is given by addition in $\mathrm{N}_{0}$. For morphisms $\sigma_{n} \in \operatorname{Aut}(\mathbf{n})$ and $\sigma_{m} \in \operatorname{Aut}(\mathbf{m})$ the automorphism $\sigma_{n} \oplus \sigma_{m}$ of $\mathbf{n}+\mathbf{m}$ is defined by applying $\sigma_{n}$ to the first $n$ elements and $\sigma_{m}$ to the last $m$ elements.

The product functor,

$$
\otimes: \Sigma \times \Sigma \rightarrow \Sigma,
$$

is defined by products of sets. Indeed, on objects it is given by multiplication in $\mathrm{N}_{0}$. We identify $\mathbf{n} \times \mathbf{m}$ with $\mathbf{n m}$ using lexicographical ordering. This means, the first $m$ elements in $\mathbf{n m}$ are identified with $\{1\} \times \mathbf{m}$, the next $m$ elements with $\{2\} \times \mathbf{m}$, etc. On morphisms, $\otimes$ is defined by the product permutation using this identification.

These operations are strictly associative and have strict units. They are also strictly symmetric (commutative) on the level of objects, but not on the induced morphisms. However; choosing the obvious permutations

$$
\underline{\mathrm{c}}_{\mathbf{n}, \mathbf{m}}^{\oplus}: \mathbf{n}+\mathbf{m} \rightarrow \mathbf{m}+\mathbf{n} \quad \text { and } \quad \underline{\mathrm{c}}_{\mathbf{n}, \mathbf{m}}^{\otimes}: \mathbf{n m} \rightarrow \mathbf{m n}
$$

as coherency isomorphisms, it is well-known that we get the structure of a bipermutative category (see e.g. [9]).

The category $\Sigma$ is an initial object in the category of bipermutative categories.

Definition 2.2. Let $\mathscr{V}$ be the topological category with

- one object $\mathrm{C}^{n}$ for each $n \in \mathrm{N}_{0}$, and

- morphisms the linear automorphisms of $C^{n}$ with the usual topology.

The direct sum functor, $\oplus$, is defined by identifying $C^{n} \oplus C^{m}$ with $C^{n+m}$ in the standard way. The tensor product functor, $\otimes$ is defined by identifying $C^{n} \otimes C^{m}$ with $\mathrm{C}^{n m}$ by using the lexicographical ordering. That is, we identify

$$
e_{1} \otimes e_{1}^{\prime}, \ldots, e_{1} \otimes e_{m}^{\prime}, e_{2} \otimes e_{1}^{\prime}, \ldots, e_{n} \otimes e_{m}^{\prime}
$$

with the standard basis in $\mathrm{C}^{n m}$, where $e_{1}, \ldots, e_{n}$ and $e_{1}^{\prime}, \ldots, e_{m}^{\prime}$ are the standard bases for $\mathrm{C}^{n}$ and $\mathrm{C}^{m}$ respectively. Both operations are strictly associative with 
units. The choices involved in identifying the bases are the same as the choices made for the elements in $\Sigma$. So, using the coherency isomorphisms from $\Sigma$ on the basis elements induces linear coherency isomorphisms for $\mathscr{V}$, making it bipermutative.

The category $\mathscr{V}$ is equivalent as a symmetric bimonoidal category to the category of finite dimensional complex vector spaces and isomorphisms. By construction we have a canonical bipermutative functor

$$
i_{\mathscr{V}}: \Sigma \rightarrow \mathscr{V} \text {. }
$$

Definition 2.3. Let $\mathscr{L}$ be the topological category with

- one object $\mathrm{C}_{n}=\mathrm{C}$ for each $n \in \mathrm{Z}$, and

- morphisms the linear automorphisms $\mathrm{C}_{n}^{*}=\mathrm{C}^{*}$ with the usual topology.

We identify the total set of morphisms with $Z \times C^{*}$ in the obvious way. Then the direct sum functor is defined by

$$
(n, a) \oplus(m, b)=(n+m, a b) .
$$

The tensor functor is defined by

$$
(n, a) \otimes(m, b)=\left(n m, a^{m} b^{n}\right) .
$$

For the sum we define the coherency twist

$$
\underline{\mathbf{c}}_{n, m}^{\oplus}=\left(n+m,(-1)^{n m}\right): \mathrm{C}_{n} \oplus \mathrm{C}_{m} \rightarrow \mathrm{C}_{m} \oplus \mathrm{C}_{n} .
$$

For the product we define the coherency twist

$$
\underline{\mathrm{c}}_{n, m}^{\otimes}=\left(n m,(-1)^{\frac{n(n-1) m(m-1)}{4}}\right): \mathrm{C}_{n} \otimes \mathrm{C}_{m} \rightarrow \mathrm{C}_{m} \otimes \mathrm{C}_{n} .
$$

As the following lemma shows this makes $\mathscr{L}$ a bipermutative category.

Both products in $\mathscr{L}$ are strictly associative and symmetric (commutative). So, the coherency isomorphisms could have been chosen to be identities. However, that is not the structure we will need. In light of this it is convenient to introduce the following terminology. Let $T: \mathscr{C} \times \mathscr{C} \rightarrow \mathscr{C} \times \mathscr{C}$ be the usual twist functor.

Definition 2.4. A monoidal category $(\mathscr{C}, \bullet)$ is called weakly strict symmetric if the functors $\bullet \mathscr{C} \times \mathscr{C} \rightarrow \mathscr{C}$ and $\bullet \circ T: \mathscr{C} \times \mathscr{C} \rightarrow \mathscr{C}$ are equal.

The term strict is, as usual, reserved for the case where the coherency isomorphism is the identity. Tautologically strict implies weakly strict. The category $\mathscr{L}$ is a weakly strict symmetric monoidal category. 
It was noted by John Rognes that there are only two possible $E_{\infty}$-ring structures on the topological space $\mathrm{Z} \times B U(1) \simeq \mathrm{Z} \times K(\mathrm{Z}, 2)$. The non-trivial of these arise as the geometric realization of $\mathscr{L}$ with the structure we defined above, and had we defined the coherency isomorphisms in $\mathscr{L}$ to be identities we would get the trivial $E_{\infty}$-ring structure.

LeMma 2.5. The category $\mathscr{L}$ is bipermutative.

Proof. Since $C^{*}$ is Abelian, $f \oplus g=g \oplus f$, and $f \otimes g=g \otimes f$ the diagrams
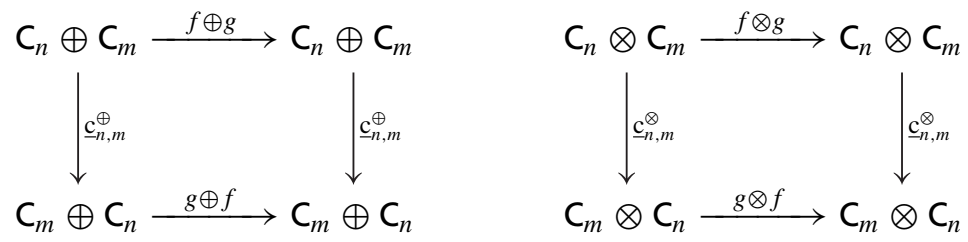

commute. So, we need to check that the diagrams spelled out in [7] involving $\underline{\mathbf{c}}_{n, m}^{\oplus}$ and $\underline{\mathbf{c}}_{n, m}^{\otimes}$ commute (we will not need the diagrams explicitly).

Let $\mathscr{L}_{+}$be the full sub-category of $\mathscr{L}$ defined by the non-negatively indexed objects. Define the functor

$$
i_{\mathscr{L}_{+}}: \Sigma \rightarrow \mathscr{L}_{+}
$$

by the obvious bijection on objects and by taking the sign of the permutation on morphisms. This preserves sums, tensor products, and coherency isomorphisms. Notice in particular the importance of the signs in Equation (5) and Equation (6). In $\Sigma$ the diagrams in question commutes ( $\Sigma$ is bipermutative). So, because $i_{\mathscr{L}_{+}}$is a bijection on objects we see that $\mathscr{L}_{+}$is bipermutative.

The coherency signs in $\mathscr{L}$ for any coherency isomorphism only depend on the objects indices modulo 4 . So, the extension of the coherency isomorphisms in $\mathscr{L}_{+}$to negatively indexed objects in $\mathscr{L}$ (using the same formulas) will still be bipermutative.

Define the functor

$$
\Lambda: \mathscr{V} \rightarrow \mathscr{L}
$$

by $C^{n} \mapsto C_{n}$ on objects and by taking determinants of morphisms. This preserves both sum and product because the determinant satisfies

$$
\operatorname{det}(f \oplus g)=\operatorname{det}(f) \operatorname{det}(g)
$$

and

$$
\operatorname{det}(f \otimes g)=\operatorname{det}(f)^{\operatorname{dim}(g)} \operatorname{det}(g)^{\operatorname{dim}(f)} .
$$


Here $\operatorname{dim}(f)$ is the dimension of the underlying vector space. The latter can be proved using $(f \otimes g)=(f \otimes \mathrm{Id}) \circ(\mathrm{Id} \otimes g)$. This explains our definition of sum and product in $\mathscr{L}$. This is a bipermutative functor because it takes coherency isomorphisms to coherency isomorphisms. Indeed, the sign of a permutation is the determinant of the associated permutation matrix, which explains why we chose the non-trivial coherency isomorphisms in $\mathscr{L}$.

We will not use the following in our construciton, but it is very helpful to keep in mind. Indeed, it tells us that $\mathscr{L}$ may be thought of as a categorical model for the second Postnikov section of the group completion of $\mathscr{V}$ with respect to $\oplus$. Note that it is very difficult to group-complete a general bipermutative category, with respect to the sum, while simultaneously retaining both products. However, this is precisely what is carried out in [2] and what we denoted by $\overline{\mathscr{V}}$ in the introduction, but we will not use this here.

LEMMa 2.6. The induced map on group-completed classifying spaces

$$
\Omega B|\Lambda|: k u \rightarrow \Omega B|\mathscr{L}| \simeq|\mathscr{L}| \simeq \mathrm{Z} \times K(\mathrm{Z}, 2),
$$

is the projection to the second Postnikov section in the category of $\infty$-loop spaces.

SKETCH OF PROOF. The geometric realizations has infinite loop space structures induced by the symmetric monoidal structure $\oplus$ and since $\Lambda$ is symmetric monoidal with respect to $\oplus$ it cannonically has the structure of an infinite loop map. So all we need to check is that it is a $\pi_{k}$-equivalence for $k \leq 2$.

The functor $\Lambda$ sends objects $N_{0}$ to $Z$ by the standard inclusion. So, we need only check that the connectivity of the map is at least three on the components corresponding to $n \in \mathrm{N}_{0}$, for large enough $n$. This corresponds to being at least 2-connected on the space of automorphisms for large $n$. The determinant

$$
\mathrm{Gl}_{n}(\mathrm{C}) \rightarrow \mathrm{C}^{*}
$$

satisfies this for all $n \geq 1$.

\section{Matrices and Determinants of Bipermutative Categories}

In this section, we define the monoidal category $M_{n}(\mathscr{C})$ of matrices in a bipermutative category $\mathscr{C}$. In the case of $\mathscr{C}=\mathscr{L}$ we define the sub-monoidal category $\mathrm{Gl}_{n}(\mathscr{L})$ of invertible objects. We then use this and the functor $\Lambda$ from Equation (7) to define the sub-monoidal category $\mathrm{Gl}_{n}(\mathscr{V})$ of weakly invertible objects in $\mathrm{M}_{n}(\mathscr{V})$. Furthermore, we construct a product preserving determinant-like functor from $\mathrm{Gl}_{n}(\mathscr{V})$ to $\mathscr{L}$, which we will see is actually not monoidal. 
Definition 3.1. For any bipermutative category $\mathscr{C}$ define $\mathrm{M}_{n}(\mathscr{C})$ as the category with

- objects $n$ by $n$ matrices $E=\left(E_{i j}\right)_{i, j=1}^{n}$ of objects $E_{i j}$ in $\mathscr{C}$ and

- morphisms $n$ by $n$ matrices $\phi=\left(\phi_{i j}\right)_{i, j=1}^{n}$ of morphisms $\phi_{i j}$ in $\mathscr{C}$ (with the obvious source and target maps).

We define a monoidal product

$$
\therefore \mathrm{M}_{n}(\mathscr{C}) \times \mathrm{M}_{n}(\mathscr{C}) \rightarrow \mathrm{M}_{n}(\mathscr{C})
$$

by basically copying the standard matrix multiplication formula:

$$
(E \cdot F)_{i k}=\bigoplus_{j=1}^{n}\left(E_{i j} \otimes F_{j k}\right) .
$$

We need not specify parentheses because $\oplus$ is strictly associative. There are obvious associativity coherency isomorphisms (from now on called associators) $\underline{\alpha}=\underline{\alpha}_{A, B, C}$ induced from the coherency isomorphisms in $\mathscr{C}$. These make $\mathrm{M}_{n}(\mathscr{C})$ a monoidal category - with a strict unit (c.f. [5]).

Notice that this category has a natural topology induced from any topology on $\mathscr{C}$.

REMARK 3.2. We could similarly define the sum of matrices and get a bimonoidal category, but this is not used in the following.

Let

$$
\Lambda_{*}: \mathrm{M}_{n}(\mathscr{V}) \rightarrow \mathrm{M}_{n}(\mathscr{L})
$$

denote the functor induced by $\Lambda$. This is a strict monoidal functor because $\Lambda$ is a bipermutative functor.

Let

$$
\operatorname{det} \mathscr{L}: \mathrm{M}_{n}(\mathscr{L}) \rightarrow \mathscr{L}
$$

be given on the total morphisms space of $\mathrm{M}_{n}(\mathscr{L})$ by taking the determinant using $\oplus$ and $\otimes$ with coefficients in $\mathscr{L}$. This is well-defined because the total morphism space of $\mathscr{L}$ with $\oplus$ and $\otimes$ can be identified with a commutative ring structure on $\mathbf{Z} \times \mathrm{C}^{*}$ (Definition 2.3). The following lemma tells us that $\operatorname{det} \mathscr{L}$ defines a functor. However, Lemma 3.7 tells us that it is not monoidal. This may be slightly surprising since by construction it preserves products.

Lemma 3.3. The map

$$
\operatorname{det} \mathscr{L}: \mathrm{M}_{n}(\mathscr{L}) \rightarrow \mathscr{L}
$$

defines a functor. 
Proof. To see that it is a functor we calculate directly. Let $D=\left(D_{i j}\right)_{i, j=1}^{n}$ be any matrix in $M_{n}(\mathrm{Z})$. Let $\left(\phi_{i j}\right)$ be an automorphism in $\mathrm{M}_{n}(\mathscr{L})$ of the corresponding matrix object $\left(C_{D_{i j}}\right)$. Then we calculate

$$
\operatorname{det} \mathscr{L}\left(\left(C_{d_{i j}}, \phi_{i j}\right)\right)=\left(C_{\operatorname{det}\left(d_{i j}\right)}, \prod_{i, j=1}^{n} \phi_{i j}^{C_{i j}}\right),
$$

where $C$ is the matrix of cofactors of $D$. Recall that we can only compose morphisms in $\mathrm{M}_{n}(\mathscr{L})$ when the object matrices agree. So, all the cofactors are the same for such. The lemma now follows because composition in $\mathscr{L}$ is given by product on the second factor and composition in $\mathrm{M}_{n}(\mathscr{L})$ is also given by product on the second factor in each entry.

Define

$$
\operatorname{det}_{\mathscr{V}}=\operatorname{det}_{\mathscr{L}} \circ \Lambda_{*}: \mathrm{M}_{n}(\mathscr{V}) \rightarrow \mathscr{L} .
$$

This functor preserves products because both $\operatorname{det}_{\mathscr{L}}$ and $\Lambda_{*}$ do so. However, again Lemma 3.7 will tell us that it is not monoidal.

Definition 3.4. Define $\mathscr{L}^{*}$ to be the full subcategory of $\mathscr{L}$ with objects $\mathrm{C}_{-1}$ and $\mathrm{C}_{1}$.

Using the identification in Definition 2.3 we see

$$
\operatorname{Mor}\left(\mathscr{L}^{*}\right)=\{ \pm 1\} \times C^{*} .
$$

This is obviously a permutative category with respect to $\otimes$. Since the twist for $\otimes$ on the object pair $\left(C_{-1}, C_{1}\right)$ is not the identity we still retain part of the non-trivial coherency structure from $\mathscr{L}$ in $\mathscr{L}^{*}$.

Definition 3.5. Define $\mathscr{L}_{+}^{*}$ to be the full sub-category of $\mathscr{L}$ with the single object $\mathrm{C}_{1}$.

Definition 3.6. Let $\mathrm{Gl}_{n}(\mathscr{V})$ be the full sub-category of $\mathrm{M}_{n}(\mathscr{V})$ defined by the pre-image of $\mathscr{L}^{*}$ using the the functor $\operatorname{det}$. Similarly, we define $\mathrm{Gl}_{n}(\mathscr{L})$ to be the pre-image of $\mathscr{L}^{*}$ using the functor $\operatorname{det} \mathscr{L}$.

One may similarly define $\operatorname{Sl}_{n}(\mathscr{V})$ and $\mathrm{Sl}_{n}(\mathscr{L})$ as the pre-images of $\mathscr{L}_{+}^{*}$. The definitions imply that $\Lambda_{*}$ maps $\mathrm{Gl}_{n}(\mathscr{V})$ to $\mathrm{Gl}_{n}(\mathscr{L})$, and similarly for $\mathrm{Sl}_{n}(\mathscr{V})$ and $\operatorname{Sl}_{n}(\mathscr{L})$.

Objects in $\mathrm{Gl}_{n}(\mathscr{V})$ are called weakly invertible matrices. The objects in $\mathrm{Gl}_{n}(\mathscr{L})$ are invertible. The definition of $\mathrm{Gl}_{n}(\mathscr{V})$ is equivalent to the definition in [5]. Indeed, the image object in $\mathscr{L}$ of $\operatorname{det} \gamma$ is by Equation (10) the determinant of the dimension matrix. So, we conclude that landing in $\mathscr{L}^{*}$ coresponds to 
having determinant of the dimension matrix equal to \pm 1 , which is equivalent to having an invertible dimension matrix.

Note that the categories $\mathrm{Gl}_{1}(\mathscr{V})$ and $\mathscr{L}_{+}^{*}$ are isomorphic. Indeed, the functor $\operatorname{det}_{\mathscr{V}}$ is an isomorphism of symmetric monoidal categories.

Lemma 3.7. For $n>1$ the functors $\operatorname{det}_{\mathscr{V}}$ and $\operatorname{det} \mathscr{L}$ are not monoidal (even when restricted to the weakly invertible matrices).

REMARK 3.8. The conclusion of Lemma 3.7 is very important and, as we will see in Section 5, it is what turns into the need for orientations on 2-vector bundles. It seems highly related to the Grassmann invariant (see [8]).

Proof. A monoidal functor has to preserve the associators. The category $\mathscr{L}$ is strictly monoidal so the associators are all identities. We will describe an associator in $\mathrm{Gl}_{n}(\mathscr{L})$, which is sent to $-1 \in \operatorname{Aut}\left(\mathrm{C}_{-1}\right)$ by $\operatorname{det} \mathscr{L}$. This will satisfy that the entries are objects $C_{n}$ for $n \geq 0$. So, it will also prove the lemma for $\operatorname{det}_{\mathscr{V}}$ because $\Lambda_{*}$ sends some associator in $\mathrm{Gl}_{n}(\mathscr{V})$ to this associator.

An example of this producing a minus sign for $n=2$ is

$$
\underline{\alpha}:\left(\left[\begin{array}{ll}
1 & 1 \\
0 & 1
\end{array}\right] \cdot\left[\begin{array}{ll}
0 & 1 \\
1 & 1
\end{array}\right]\right) \cdot\left[\begin{array}{ll}
1 & 0 \\
1 & 1
\end{array}\right] \rightarrow\left[\begin{array}{ll}
1 & 1 \\
0 & 1
\end{array}\right] \cdot\left(\left[\begin{array}{ll}
0 & 1 \\
1 & 1
\end{array}\right] \cdot\left[\begin{array}{ll}
1 & 0 \\
1 & 1
\end{array}\right]\right),
$$

where $k=\mathrm{C}_{k}$ in $\mathscr{L}$. This is an automorphism of the object

$$
\left[\begin{array}{ll}
3 & 2 \\
2 & 1
\end{array}\right]
$$

To see which one, we decorate the entries, and calculate:

$$
\begin{aligned}
\left(\left[\begin{array}{ll}
1_{1} & 1_{2} \\
0 & 1_{3}
\end{array}\right] \cdot\left[\begin{array}{cc}
0 & 1_{a} \\
1_{b} & 1_{c}
\end{array}\right]\right) \cdot\left[\begin{array}{cc}
1_{\alpha} & 0 \\
1_{\beta} & 1_{\gamma}
\end{array}\right] \\
=\left[\begin{array}{cc}
1_{2 b \alpha} \oplus 1_{1 a \beta} \oplus 1_{2 c \beta} & 1_{1 a \gamma} \oplus 1_{2 c \gamma} \\
1_{3 b \alpha} \oplus 1_{3 c \beta} & 1_{3 c \gamma}
\end{array}\right], \\
{\left[\begin{array}{cc}
1_{1} & 1_{2} \\
0 & 1_{3}
\end{array}\right] \cdot\left(\left[\begin{array}{cc}
0 & 1_{a} \\
1_{b} & 1_{c}
\end{array}\right] \cdot\left[\begin{array}{cc}
1_{\alpha} & 0 \\
1_{\beta} & 1_{\gamma}
\end{array}\right]\right) } \\
=\left[\begin{array}{cc}
1_{1 a \beta} \oplus 1_{2 b \alpha} \oplus 1_{2 c \beta} & 1_{1 a \gamma} \oplus 1_{2 c \gamma} \\
1_{3 b \alpha} \oplus 1_{3 c \beta} & 1_{3 c \gamma}
\end{array}\right] .
\end{aligned}
$$

Here $1_{2 b \alpha}=1_{2} \otimes 1_{b} \otimes 1_{\alpha}$ and similarly for the other terms. We used here that the product of 0 with anything is strictly 0 , and that 0 is a strict unit for $\oplus$. We also used that 1 is a strict unit for $\otimes$, which implies that when either the left or right distributive law is used in the calculation it is strict. We now see that the associator is a twist isomorphism for $\oplus$ in the first two factors of the 
$(1,1)$ entry. This twist isomorphism is $-1 \in \operatorname{Aut}\left(C_{2}\right)$. So, the total morphism in entry $(1,1)$ is $(-1) \in \operatorname{Aut}\left(C_{3}\right)$. The associator is the identity in all other entries and so

$$
\operatorname{det}_{\mathscr{L}}(\underline{\alpha})=\left(1 \cdot 3-2 \cdot 2, \frac{1^{3}(-1)^{1}}{1^{2} 1^{2}}\right)=(-1,-1) \in \mathrm{Z} \times \mathrm{C}^{*}=\operatorname{Mor}(\mathscr{L}) .
$$

This example also works for larger $n$ by doing block sum with identity matrices. The block sum functor is described in more detail in Section 6.

For $n=4$ one may take block sum with the identity on two of the three matrices above and block sum with

$$
\left[\begin{array}{ll}
0 & 1 \\
1 & 0
\end{array}\right]
$$

on the last. This produces an example in which the associator $\underline{\alpha}$ is sent to an automorphism of $\mathrm{C}_{1}$ and not of $\mathrm{C}_{-1}$. This shows that even restricting to $\mathrm{Sl}_{n}(\mathscr{V})$ does not make $\operatorname{det} \gamma$ monoidal for $n \geq 4$.

\section{Orientations on the Category Level}

In this Section, we "enlarge" the categories $\mathrm{Gl}_{n}(\mathscr{V})$ and $\mathrm{Gl}_{n}(\mathscr{L})$ to oriented versions $\mathrm{O}\left(\mathscr{V}^{n}\right)$ and $\mathrm{O}\left(\mathscr{L}^{n}\right)$. On these it is possible to define monoidal determinant-like maps to the monoidal category $\left(\mathscr{L}^{*}, \otimes\right)$. We then construct a monoidal functor

$$
\mathscr{G}: \mathrm{O}\left(\mathscr{V}^{n}\right) \rightarrow \mathscr{L}_{+}^{*} \cong \mathrm{Gl}_{1}(\mathscr{V}),
$$

which we use in Section 6 to (re-)construct gerbes from oriented 2-vector bundles.

As a category we define $\mathrm{O}\left(\mathscr{V}^{n}\right)$ by

$$
\mathrm{O}\left(\mathscr{V}^{n}\right)=\mathrm{Gl}_{n}(\mathscr{V}) \times B \mathrm{Z}^{*} .
$$

Here $B Z^{*}$ is the category with one object and automorphisms $Z^{*}=\{ \pm 1\}$. The category $B Z^{*}$ is strictly monoidal by defining the product to be equal to the composition. The monoidal product on $\mathrm{O}\left(\mathscr{V}^{n}\right)$ (disregarding the associators) is defined coordinate-wise. However, we will "lift" the associators in the following non-trivial way.

Let $\varphi$ be any morphism in $\mathrm{Gl}_{n}(\mathscr{V})$ such that $\operatorname{det}_{\mathscr{V}}(\varphi)=( \pm 1, \pm 1)$ (using the identification of $\left.\operatorname{Mor}(\mathscr{L})=Z \times C^{*}\right)$. Define $\operatorname{sgn}(\varphi)$ to be the sign in the last factor (i.e. the morphism part of $\operatorname{det}_{\mathscr{V}}(\varphi)$ ). The sign satisfies

- $\operatorname{sgn}\left(\varphi_{1} \circ \varphi_{2}\right)=\operatorname{sgn}\left(\varphi_{1}\right) \operatorname{sgn}\left(\varphi_{2}\right)$,

- $\operatorname{sgn}\left(\varphi_{1} \cdot \varphi_{2}\right)=\operatorname{sgn}\left(\varphi_{1}\right) \operatorname{sgn}\left(\varphi_{2}\right)$, and 
- $\operatorname{sgn}\left(\operatorname{Id}_{A}\right)=1$ for all objects $A$ in $\mathrm{Gl}_{n}(\mathscr{V})$.

The first and the last follow from Lemma 3.3. The second because $\operatorname{det}_{\mathscr{V}}$ preserves products and

$$
\begin{aligned}
\operatorname{det}_{\mathscr{V}}\left(\varphi_{1} \cdot \varphi_{2}\right) & =\operatorname{det}_{\mathscr{V}}\left(\varphi_{1}\right) \otimes \operatorname{det}_{\mathscr{V}}\left(\varphi_{2}\right) \\
& =\left( \pm 1, \operatorname{sgn}\left(\varphi_{1}\right)^{ \pm 1} \operatorname{sgn}\left(\varphi_{2}\right)^{ \pm 1}\right) \\
& =\left( \pm 1, \operatorname{sgn}\left(\varphi_{1}\right) \operatorname{sgn}\left(\varphi_{2}\right)\right) .
\end{aligned}
$$

Here we critically used that $\operatorname{sgn}\left(\varphi_{i}\right)= \pm 1$.

For any associator $\underline{\alpha}$ in $\operatorname{Gl}_{n}(\mathscr{V})$ the $\operatorname{sign} \operatorname{sgn}(\underline{\alpha})$ is defined. Indeed, all coherency isomorphisms in $\mathscr{V}$ have determinant \pm 1 , and all sums, products and compositions of such in $\mathscr{L}$ yield a sign. Using sgn we thus define the associators $\underline{\alpha}^{\prime}$ of $\mathrm{O}\left(\mathscr{V}^{n}\right)$ by

$$
\underline{\alpha}_{A, B, C}^{\prime}=\left(\underline{\alpha}_{A, B, C}, \operatorname{sgn}\left(\underline{\alpha}_{A, B, C}\right)\right) .
$$

Here $\underline{\alpha}_{A, B, C}$ is the corresponding associator in $\mathrm{Gl}_{n}(\mathscr{V})$. Using that the pentagon axiom in $\mathrm{Gl}_{n}(\mathscr{V})$ is satisfied, and using the above properties of sgn, it is easy to see that the diagram

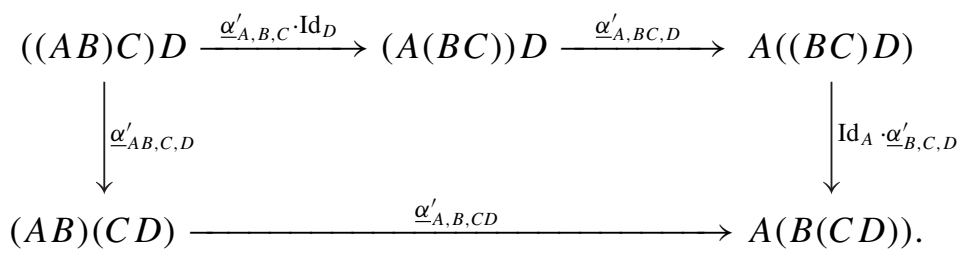

commutes. Thus the pentagon axiom is also satisfied in $\mathrm{O}\left(\mathscr{V}^{n}\right)-$ making it monoidal.

We may define $\mathrm{O}\left(\mathscr{L}^{n}\right)$ completely analogously using $\operatorname{det} \mathscr{L}$. We may also define "lifted" functors

$$
\begin{aligned}
\operatorname{det}_{\mathscr{L}}^{\text {or }}: \mathrm{O}\left(\mathscr{L}^{n}\right) & \rightarrow \mathscr{L}^{*}, \\
\operatorname{det}_{\mathscr{V}}^{\text {or }}: \mathrm{O}\left(\mathscr{V}^{n}\right) & \rightarrow \mathscr{L}^{*}, \quad \text { and } \\
\Lambda_{*}^{\text {or }}: \mathrm{O}\left(\mathscr{V}^{n}\right) & \rightarrow \mathrm{O}\left(\mathscr{L}^{n}\right),
\end{aligned}
$$

defined on morphisms by

$$
\begin{aligned}
\operatorname{det}_{\mathscr{L}}^{\text {or }}(f, s) & =\operatorname{det}_{\mathscr{L}}(f) \otimes(1, s), \\
\operatorname{det}_{\mathscr{V}}^{\text {or }}(g, s) & =\operatorname{det}_{\mathscr{V}}(g) \otimes(1, s), \quad \text { and } \\
\Lambda_{*}^{\text {or }}(g, s) & =\left(\Lambda_{*}(g), s\right) .
\end{aligned}
$$


The result of tensoring with $(1, s)$ is multiplication with the sign $s$ on the morphism in $\mathscr{L}^{*}$. This is true only in $\mathscr{L}^{*}$, not in all of $\mathscr{L}$. So, it is in fact very important that we have restricted to the weakly invertible matrices. These oriented functors preserve products since the unoriented did and $\otimes$ is weakly strictly symmetric in $\mathscr{L}$. The point of the construction is that $\operatorname{det}_{\mathscr{L}}^{\text {or }}$ and $\operatorname{det}_{\mathscr{V}}^{\text {or }}$ are in fact strict monoidal. Indeed, the signs of the associators $\underline{\alpha}^{\prime}$ were chosen such that they cancel with the sign that made $\operatorname{det} \mathscr{L}$ and $\operatorname{det}_{\gamma}$ not be monoidal.

COROLLARY 4.1. The functors $\operatorname{det}_{\mathscr{V}}^{\text {or }}$ and $\operatorname{det}_{\mathscr{L}}^{\text {or }}$ are strictly monoidal.

There are canonical strict monoidal functors:

$$
\begin{aligned}
& P_{\mathscr{V}}: \mathrm{O}\left(\mathscr{V}^{n}\right) \rightarrow \mathrm{Gl}_{n}(\mathscr{V}) \\
& P_{\mathscr{L}}: \mathrm{O}\left(\mathscr{L}^{n}\right) \rightarrow \mathrm{Gl}_{n}(\mathscr{L}),
\end{aligned}
$$

defined by forgetting the sign.

Remark 4.2. The composites of $P_{\mathscr{L}}$ and $P_{\mathscr{V}}$ with $\operatorname{det}_{\mathscr{L}}$ and $\operatorname{det}_{\mathscr{V}}$ respectively are not the oriented functors. Indeed, if we forget the sign we cannot multiply by it. However, the diagram

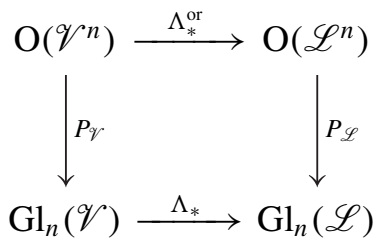

is obviously a commutative diagram of monoidal functors.

Later when we want to construct gerbes we need a determinant-like map landing in $\mathscr{L}_{+}^{*}$, and not in $\mathscr{L}$. So, consider the inclusion

$$
\mathscr{L}_{+}^{*} \rightarrow \mathscr{L}^{*},
$$

which is a strict symmetric monoidal functor. We have a left inverse (or projection)

$$
p: \mathscr{L}^{*} \rightarrow \mathscr{L}_{+}^{*}
$$

given by $p(d, a)=\left(1, a^{d}\right)$. This is not symmetric monoidal because the symmetry on -1 is not the identity. It is, however, strict monoidal because

$$
\begin{aligned}
p((d, a) \otimes(e, b)) & =p\left(d e, a^{e} b^{d}\right)=\left(1, a^{d} b^{e}\right) \\
& =\left(1, a^{d}\right) \otimes\left(1, b^{e}\right) \\
& =p((d, a)) \otimes p((e, b)),
\end{aligned}
$$


and all associators are identities. We thus define the strict monoidal functor $\mathscr{G}$ by the composition

$$
\mathscr{G}=\left(p \circ \operatorname{det}_{\mathscr{V}}^{\text {or }}\right): \mathrm{O}\left(\mathscr{V}^{n}\right) \rightarrow \mathscr{L}_{+}^{*} \cong \mathrm{Gl}_{1}(\mathscr{V})
$$

\section{Orientations on 2-Vector Bundles}

In this section, we recall the definition of a charted 2-vector bundle on a topological space $X$. We then use the construction in Section 4 to define oriented charted 2-vector bundles. Then we describe the precise obstruction for a 2vector bundle to be oriented. This turns out to be a characteristic class in the third cohomology group of $X$ (for well-behaved $X$ ).

Some of the following definitions are taken from [5] and [3]. In the following $X$ is a topological space.

Definition 5.1. An ordered open cover $(\mathscr{U}, \mathscr{J})$ of $X$ is a collection $\mathscr{U}=$ $\left\{U_{\alpha} \mid \alpha \in \mathscr{J}\right\}$ of non-empty open sets $U_{\alpha} \subset X$, indexed by a partially ordered set $\mathscr{J}$, such that

- the $U_{\alpha}$ cover $X$, and

- the partial ordering on $\mathscr{J}$ restricts to a total ordering on each finite subset $\left\{\alpha_{0}, \ldots, \alpha_{p}\right\}$ of $\mathscr{J}$ for which the intersection $U_{\alpha_{0} \ldots \alpha_{p}}=U_{\alpha_{0}} \cap \cdots \cap U_{\alpha_{p}}$ is nonempty.

As noted before there are induced topologies on the category $\mathrm{M}_{n}(\mathscr{V})$, the category $\mathrm{M}_{n}(\mathscr{L})$, the category $\mathrm{Gl}_{n}(\mathscr{V})$, the category $\mathrm{Gl}_{n}(\mathscr{L})$, the category $\mathrm{O}\left(\mathscr{V}^{n}\right)$, and the category $\mathrm{O}\left(\mathscr{L}^{n}\right)$. The objects in each of these form a discrete space. Furthermore, all structures and functors constructed are easily seen to be continuous. Let $(\mathscr{C}, \cdot)$ be a topological monoidal category with discrete objects.

Definition 5.2. A principal $\mathscr{C}$-bundle $\mathscr{E}$ on $X$ is

1) an ordered open cover $(\mathscr{U}, \mathscr{J})$ of $X$,

2) for each $\alpha<\beta$ in $\mathscr{J}$ an object $E^{\alpha \beta}$ in $\mathscr{C}$, such that for each $\alpha<\beta<\gamma$ we have

$$
E^{\alpha \beta} \cdot E^{\beta \gamma}=E^{\alpha \gamma}
$$

on the level of objects, and

3) for each $\alpha<\beta<\gamma$ we have maps

$$
\phi^{\alpha \beta \gamma}: U_{\alpha \beta \gamma} \rightarrow \operatorname{Mor}\left(E^{\alpha \beta} \cdot E^{\beta \gamma}, E^{\alpha \gamma}\right),
$$

called the coherency maps such that 
4) the diagram

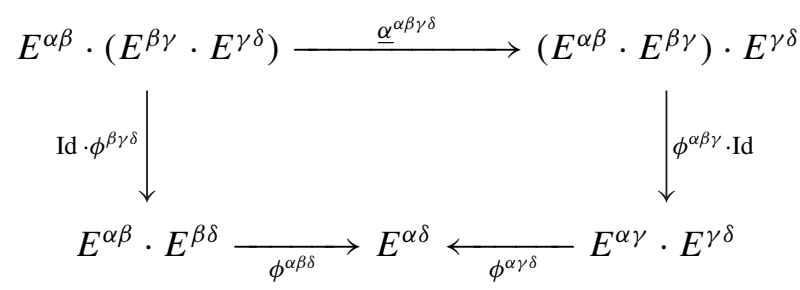

commutes for all points in each quadruple intersection $U_{\alpha \beta \gamma \delta}$.

Here $\underline{\alpha}^{\alpha \beta \gamma \delta}$ denotes the associator for the product - in $\mathscr{C}$ related to the two different choices of parentheses. The diagram may be thought of as a cocycle condition.

Note that the definition of principal $\mathscr{C}$-bundles is obviously functorial with respect to continuous strict monoidal functors.

Definition 5.3. A charted 2-vector bundle $\mathscr{E}$ of rank $n \in \mathrm{N}$ over $X$ is a principal $\mathrm{Gl}_{n}(\mathscr{V})$-bundle. An oriented charted 2-vector bundle $\mathscr{E}$ of rank $n$ over $X$ is a principal $\mathrm{O}\left(\mathscr{V}^{n}\right)$-bundle.

This is slightly different from the definition in [5]. However, in the unoriented case the definition is equivalent in the following sense.

Definition 5.4. Two (oriented) charted 2-vector bundles $\mathscr{E}_{i}, i=0,1$ over $X$ are equivalent if they are cobordant. I.e., if there exists a (oriented) charted 2 -vector bundle $\mathscr{E}$ over $X \times[0,1]$ such that $\mathscr{E}_{\mid X \times\{i\}}=\mathscr{E}_{i}$ for $i=0$, 1 . Here restriction of the ordered open cover removes the sets (and their indices) which have empty intersection with $X \times\{i\}$.

We use Definition 5.4, which is from [3], as opposed to the alternative in [5]. Indeed, it is easier to work with. However, it is less explicit.

Recall the functor $P_{\mathscr{V}}$ from Equation (12).

Definition 5.5. A charted 2-vector bundle is said to be orientable if it is equivalent to $P_{\mathscr{V}}(\mathscr{E})$, where $\mathscr{E}$ is an oriented charted 2-vector bundle.

Lemma 5.6. For a charted 2-vector bundle $\mathscr{E}$ on an ordered open cover $(\mathscr{U}, \mathscr{J})$ the signs

$$
\operatorname{sgn}\left(\underline{\alpha}^{\alpha \beta \gamma \delta}\right), \quad \alpha<\beta<\gamma<\delta \in \mathscr{J}
$$

of the associators define a 3-cocycle in the Čech complex $\check{C}^{*}(\mathcal{U},\{ \pm 1\})$. The represented class in Čech cohomology only depends on the equivalence class of the 2-vector bundle. Furthermore, this class is zero if and only if the vector bundle is orientable. 
The following proof is very similar to the arguments in Section 4 .

Proof. The Lane-Stasheff pentagon axiom tells us that

$$
\underline{\alpha}^{\alpha \gamma \delta \epsilon} \circ \underline{\alpha}^{\alpha \beta \gamma \epsilon}=\left(\underline{\alpha}^{\alpha \beta \gamma \delta} \cdot \operatorname{Id}_{E^{\delta \epsilon}}\right) \circ \underline{\alpha}^{\alpha \beta \delta \epsilon} \circ\left(\operatorname{Id}_{E^{\alpha \beta}} \cdot \underline{\alpha}^{\beta \gamma \delta \epsilon}\right) .
$$

Taking sgn, and using the properties of sgn from Section 4, we get

$$
\operatorname{sgn}\left(\underline{\alpha}^{\alpha \gamma \delta \epsilon}\right) \operatorname{sgn}\left(\underline{\alpha}^{\alpha \beta \gamma \epsilon}\right)=\operatorname{sgn}\left(\underline{\alpha}^{\alpha \beta \gamma \delta}\right) \operatorname{sgn}\left(\underline{\alpha}^{\alpha \beta \delta \epsilon}\right) \operatorname{sgn}\left(\underline{\alpha}^{\beta \gamma \delta \epsilon}\right),
$$

which is the cocycle condition. Obviously the associated homology class only depends on the equivalence class since the inclusions of $X \times\{i\}$ into $X \times[0,1]$ is a homotopy equivalence.

This class is zero if and only if there is a refinement $\left(\mathscr{U}^{\prime}, \mathscr{J}^{\prime}\right)$ of the ordered open cover and a chain $a$ in $\check{C}^{2}\left(\mathscr{U}^{\prime},\{ \pm 1\}\right)$ s.t. $\partial a=\operatorname{sgn}(\underline{\alpha})$. Furthermore, such a choice exactly corresponds to a lift of the coherency maps $\phi^{\alpha \beta \gamma}$ to $\left(\phi^{\alpha \beta \gamma}, a\right)$ in $\mathrm{O}\left(\mathscr{V}^{n}\right)$ satisfying the diagram in Equation (15).

\section{The Determinant Gerbe}

In this section, we define gerbes and their inclusion into 2-vector bundles. We then lift this to oriented 2-vector bundles and define a retraction back to gerbes. As described in the introduction such a retraction does not exist for unoriented 2 -vector bundles.

DeFinition 6.1. A charted gerbe is a charted $\mathrm{Gl}_{1}(\mathscr{V})$-bundle.

Note that $\mathrm{Gl}_{1}(\mathscr{V})$ has one object with automorphisms $\mathrm{C}^{*}$. So, this is the same as having a standard 2-cocycle with coefficients in $\mathrm{C}^{*}$. So, the definition is compatible with Brylinski's definition of a charted gerbe in [6].

The block sum construction on matrices defines the strict monoidal functor

$$
S: \mathrm{Gl}_{n}(\mathscr{V}) \times \mathrm{Gl}_{m}(\mathscr{V}) \rightarrow \mathrm{Gl}_{n+m}(\mathscr{V}) .
$$

Let

$$
\left(\mathscr{I}_{n-1}\right)_{i j}=\left\{\begin{array}{ll}
\mathrm{C}^{0} & i \neq j \\
\mathrm{C}^{1} & i=j
\end{array} .\right.
$$

be the $n-1$ by $n-1$ identity matrix in $\mathrm{Gl}_{n-1}(\mathscr{V})$ (identity object). Block sum with $\mathscr{I}_{n-1}$ thus defines a strict monoidal functor

$$
i \mathscr{G}: \mathrm{Gl}_{1}(\mathscr{V}) \rightarrow \mathrm{Gl}_{n}(\mathscr{V})
$$

Of course to define the block sum with $\mathscr{I}_{n-1}$ on morphisms we use the identity morphism on $\mathscr{I}_{n-1}$. The inclusion $i_{\mathscr{G}}$ induces the standard inclusion of gerbes into 2-vector bundles. 
The associator in $\mathrm{Gl}_{1}(\mathscr{V})$ is the associator for $\otimes$ in $\mathscr{V}$ on the object $\mathrm{C}$. This means that it is the identity and has sign 1 . The inclusion of $\mathrm{Gl}_{1}(\mathscr{V}) \rightarrow \mathrm{Gl}_{n}(\mathscr{V})$ may thus be lifted to the oriented category $\mathrm{O}\left(\mathscr{V}^{n}\right)$. Indeed, we have a strict monoidal functor

$$
i_{\mathscr{G}}^{\text {or }}: \mathrm{Gl}_{1}(\mathscr{V}) \rightarrow \mathrm{O}\left(\mathscr{V}^{n}\right),
$$

given by block summing with $\mathscr{I}_{n-1}$ and simply assigning the sign 1 to all morphisms.

In [5] and [3] (and something similar appeared in [10]) it is described how to construct a simplicial category $\mathscr{B} \mathscr{C}$ from a monoidal category $\mathscr{C}$. This has the property that the geometric realization

$$
|\mathscr{B} \mathscr{C}|
$$

of the nerve of $\mathscr{B} \mathscr{C}$ is a classifying space for equivalence classes of principal $\mathscr{C}$ bundles (for well-behaved $X$ ). There are certain conditions that $\mathscr{C}$ must satisfy, but all the categories we consider here satisfy these. It is used repeatedly in the following that $\mathscr{B}$ is a functor from the category of monoidal categories and strict monoidal functors to simplicial categories.

Since $\mathrm{Gl}_{1}(\mathscr{V})$ has one object with automorphisms $C^{*}$ it follows that $\left|\mathscr{B} \mathrm{Gl}_{1}(\mathscr{V})\right|$ is a $K(\mathrm{Z}, 3)$ and in [1] it is proven that the map

$$
\left|\mathscr{B}\left(i_{\mathscr{G}}\right)\right|_{*}: \pi_{3}\left(\left|\mathscr{B} \mathrm{Gl}_{1}(\mathscr{V})\right|\right) \rightarrow \pi_{3}\left(\left|\mathscr{B} \mathrm{Gl}_{n}(\mathscr{V})\right|\right)
$$

sends the canonical generator to an element divisible by 2 modulo torsion. Indeed, this is the magnetic monopole, which obstructs the existence of a retraction back to $\left|\mathscr{B} \mathrm{Gl}_{1}(\mathscr{V})\right| \simeq K(\mathrm{Z}, 3)$. The point of the orientations is that the monoidal functor $\mathscr{G}$ from equation (14) provides a retraction:

$$
|\mathscr{B} \mathscr{G}|:\left|\mathscr{B O}\left(\mathscr{V}^{n}\right)\right| \rightarrow\left|\mathscr{B} \mathrm{Gl}_{1}(\mathscr{V})\right| \simeq K(\mathrm{Z}, 3)
$$

of $\left|\mathscr{B} i_{\mathscr{G}}^{\text {or }}\right|$. We thus conclude that we have removed the obstruction and that any half magnetic monopole must be a non-norientable 2-vector bundle over $S^{3}$.

The following corollary is used only as motivation in the introduction. However, it follows rather nicely from the construction thus far; and it puts a nice perspective on some issues.

COROLlaRY 6.2. There is a retraction of the inclusion

$$
K(\mathrm{Z}, 3) \simeq B_{\otimes} B U(1) \rightarrow B_{\otimes}(\{ \pm 1\} \times B U) \simeq B_{\otimes}\left(k u^{*}\right) .
$$

Note that this fact is equivalent to the fact that the third Postnikov section of $B_{\otimes}\left(k u^{*}\right)$ splits as $K(\mathrm{Z} / 2,1) \times K(\mathrm{Z}, 3)$. 
SKetch of PROOF. We know that the second Postnikov section of the loop space of $B_{\otimes}\left(k u^{*}\right)$ splits as $Z / 2 \times K(Z, 2)$. Indeed, the loops space is $Z / 2 \times B U$.

By Lemma 2.6 we see that this second Postnikov section with the loop structure coming from $\otimes$ is represented by $(\mathscr{L}, \otimes)$. So since the functor $p$ from Equation (13) is monoidal with respect to $\otimes$ we get the retraction as

$$
B_{\otimes}\left(k u^{*}\right) \stackrel{P_{3}}{\longrightarrow}\left|\mathscr{B}_{\otimes}\left(\mathscr{L}^{*}\right)\right| \stackrel{\left|\mathscr{B}_{\otimes} p\right|}{\longrightarrow}\left|\mathscr{B} \mathscr{L}_{+}^{*}\right| \simeq K(\mathrm{Z}, 3),
$$

where $P_{3}$ is the third Postnikov section.

Notice that in constructing $p$ we used that the monoidal product $\otimes$ is trivial. However, it is not trivial as a symmetric monoidal product, and this is precisely why the splitting can deloop once, but we cannot assume any more deloopings.

\section{Oriented Block Sum and $K^{\text {or }}(k u)$}

In this section we generalize the block sum functor from Equation (16) to the oriented categories. We also define $K^{\text {or }}(\mathscr{V})$, which is an oriented version of $K(\mathscr{V}) \simeq K(k u)$.

To generalize the block sum functor to the oriented categories we need to incorporate the sign. So, we define the functor

$$
S^{\text {or }}: \mathrm{O}\left(\mathscr{V}^{n}\right) \times \mathrm{O}\left(\mathscr{V}^{m}\right) \rightarrow \mathrm{O}\left(\mathscr{V}^{n+m}\right)
$$

by block sum on objects, block sum on the first factor of the morphisms, and by multiplying the signs on the second factor. This is obviously a product preserving functor (by ignoring the coherencies in the definition of $\mathrm{O}\left(\mathscr{V}^{n}\right)$ ). So, if it preserves the associators it will be strictly monoidal. Since we know standard block sum to be strictly monoidal all we need is to check the signs. Again this works out because we restricted to the weakly invertible matrices. Indeed, we see that

$$
\begin{aligned}
\operatorname{det} \mathcal{V}\left(S\left(\underline{\alpha}^{n}, \underline{\alpha}^{m}\right)\right) & =\operatorname{det} \mathcal{V}\left(\underline{\alpha}^{n}\right) \otimes \operatorname{det} \mathcal{V}\left(\underline{\alpha}^{m}\right) \\
& =\left( \pm 1, \operatorname{sgn}\left(\underline{\alpha}^{n}\right)\right) \otimes\left( \pm 1, \operatorname{sgn}\left(\underline{\alpha}^{m}\right)\right) \\
& =\left( \pm 1, \operatorname{sgn}\left(\underline{\alpha}^{n}\right) \operatorname{sgn}\left(\underline{\alpha}^{m}\right)\right)
\end{aligned}
$$

for $\underline{\alpha}^{n}$ and $\underline{\alpha}^{m}$ associators in $\mathrm{Gl}_{n}(\mathscr{V})$ and $\mathrm{Gl}_{m}(\mathscr{V})$ respectively. So, the sign of the block sum of two associators is the product of the signs of the associators. So, $S^{\text {or }}$ is strictly monoidal.

We may thus define oriented K-theory of $\mathscr{V}$ as

$$
K^{\text {or }}(\mathscr{V})=\Omega B \coprod_{n \in \mathrm{N}}\left|\mathscr{B} \mathrm{O}\left(\mathscr{V}^{n}\right)\right| \simeq \mathrm{Z} \times\left|\mathscr{B} \mathrm{O}\left(\mathscr{V}^{\infty}\right)\right|^{+},
$$


where the monoid structure on $\bigsqcup_{n \in \mathrm{N}}\left|\mathscr{B O}\left(\mathscr{V}^{n}\right)\right|$ is induced by the block sum functor $S^{\text {or }}$.

\title{
REFERENCES
}

1. Ausoni, C., Dundas, B. I., and Rognes, J., Divisibility of the Dirac magnetic monopole as a two-vector bundle over the three-sphere, Doc. Math. 13 (2008), 795-801.

2. Baas, N. A., Dundas, B. I., Richter, B., and Rognes, J., Ring completion of rig categories, arXiv:math/0706.0531v4 (2010).

3. Baas, N. A., Bökstedt, M., and Kro, T. A., Two-categorical bundles and their classifying cpaces, J. K-Theory 10 (2012), 299-369.

4. Baas, N. A., Dundas, B. I., Richter, B., and Rognes, J., Stable bundles over rig categories, J. Topol. 4 (2011), 623-640.

5. Baas, N. A., Dundas, B. I., and Rognes, J., Two-vector bundles and forms of elliptic cohomology, pp. 18-45 in: Topology, geometry and quantum field theory, London Math. Soc. Lect. Note Ser. 308, Cambr. Univ. Press, Cambridge 2004.

6. Brylinski, J-L., Loop spaces, characteristic classes and geometric quantization, reprint of 1993 ed., Modern Birkhäuser Classics, Birkhäuser, Basel 2008.

7. Elmendorf, A. D., and Mandell, M. A., Rings, modules, and algebras in infinite loop space theory, Adv. Math. 205 (2006), 163-228.

8. Igusa, K., On the algebraic $K$-theory of $A_{\infty}$-ring spaces, pp. 146-194 in: Algebraic $K$-theory, Part II, Oberwolfach 1980, Lect. Notes Math. 967, Springer, Berlin 1982.

9. May, J. P., $E_{\infty}$ ring spaces and $E_{\infty}$ ring spectra, contributions by Quinn, F., Ray, N., and Tornehave, J., Lect. Notes Math. 577, Springer, Berlin 1977.

10. Thomason, R. W., First quadrant spectral sequences in algebraic $K$-theory via homotopy colimits, Comm. Algebra 10 (1982), 1589-1668.

\author{
UPPSALA UNIVERSITY \\ DEPARTMENT OF MATHEMATICS \\ P.O. BOX 480 \\ 75106 UPPSALA \\ SWEDEN \\ E-mail: thomas.kragh@math.uu.se
}

